Volume 8, No.1.6, 2019

International Journal of Advanced Trends in Computer Science and Engineering

Available Online at http://www.warse.org/IJATCSE/static/pdf/file/ijatcse8081.62019.pdf

https://doi.org/10.30534/ijatcse/2019/8081.62019

\title{
MUA3D: Malaysian Ethnicity Recognition
}

\author{
Izzah Nilamsyukriyah Binti Buang \\ Faculty of Computation Science and Information \\ Technology \\ University Malaysia Sarawak \\ Sarawak, Malaysia \\ ins6728@gmail.com
}

\author{
Hamimah Ujir \\ Faculty of Computation Science and Information \\ Technology \\ University Malaysia Sarawak \\ Sarawak, Malaysia \\ uhamimah@fit.unimas.my
}

\begin{abstract}
From a human face, one can tell that person's emotion, gender and ethnicity. The ethnicity recognition score contributes to increase the matching score in the face recognition. This paper presents Malaysian ethnicity recognition using a new face database, MUA, developed by a biometric research group, Sarawak Biometric (SARAB). This database contains data from three main ethnic groups in Malaysia mainly Malay, Chinese and Indian. In addition, MUA also contains data for the biggest ethnics in Sarawak, the Ibans or called Sea Dayaks. We used 200 face data from MUA. Several geometric features were extracted from the images. The classifier used is Support Vector Machine whereas the accuracy obtained for facial ethnicity classification is $92.01 \%$.
\end{abstract}

Key words: Face recognition, face database, ethnicity classification, support vector machines, ethnic face database

\section{INTRODUCTION}

The success in face recognition area has paved the way for age detection, facial expression and gender classification. All of these face analysis works have brought benefits to other areas such as marketing, security and can be further extended to neuroscience and social psychology studies. However, a lesser number of works on the face analysis field of study focus on ethnicity recognition. The capability to distinguish age, gender, ethnicity and emotion displayed of others is also significant for the coordination of social behavior.

As we know, face carries spacious information about a person, such as race, gender, age, expression, and identity. The studies in psychology prove that when confronting human face usually will trigger three conscious neural evaluations, which are race, gender and age. Among them, a race is said to be the most outstanding attribute to be conceived by a series of social cognitive and perceptual tasks. However, the problem arises as the computational mechanism facing complexity to classify ethnicity based on the facial features.

This paper presents a new Malaysian Face Database called MUA, and the images for this database are captured by Kinect. The second objective is to recognize ethnicity using Support Vector Machines (SVM). Section 2 describes the related works on this field, followed by a discussion on the experiment settings in section 3. Then, the results and analysis are discussed in section 4. Finally, the conclusions are drawn.

\section{RELATED WORKS}

Several researches have been carried out to solve ethnicity/race based on facial features. For example, research in psychology has deliberated for behaviour interrelations of race perception such as other-race-effect (ORE) and attention model in [1], [2], [3], [4] and [5], which portray the existence of racially-discriminative facial features. Computational neuroscientist also has produced models to trigger and describe race perception in [6], [7], [8], [9] and [10]. Experiments in [11], [12] and [13] in addition revealed a notable visual factor of racial features.

As for the process of classification of ethnicity, we should begin with the elemental clarification and perception of the ethnic group itself. Based on the Oxford dictionary, ethnicity is a state of belonging to a social group that has a common national or cultural tradition. Based on [14], ethnicity is a complex social construct that influences personal identity and group social relations and [14] states that ethnicity is defined in terms of shared genealogy.

Nonetheless, to imply the basic algorithm tends to be varied and sophisticated. Firstly, the classification of race is still puzzling by a variety of perspectives, which lead to ambiguity in formulation and methodology. Secondly, in order to construct a competitive automatic race recognition, large-scale of database needed to be trained and established. Thirdly, as nowadays 3D facial fiducial data are much more favourable for computational recognition rather than human recognition.

Early studies have been done using an appearance-based methodology, which used the basis of colour, texture and shape-based algorithms. Although it is straightforward, computationally adequate, and competent performance, these approaches are said to precede the encoding procedure of the human visual system [15]. Additionally, it will appear as a downturn results when confronting with image manipulation such as changes in scale and illumination.

Consequently, later approaches use a feature-based track and also looks at both configure and shape aspect. [16] adopt 25 measurements from a head and facial landmarks to differentiate ethnic morphology between three groups, which are North-American Caucasians, African-Americans and Chinese. In another word, this study suggesting using information of distinctive characteristics for each ethnic to enhance recognition accuracy. In 2006, [17] introduced a 
multimodal method to identify ethnicity using Support Vector Machine (SVM) using depth and texture facial data. The results show that the depth approaches is more descriptive in comparison to intensity modality for ethnicity classification. [18] then proposed a fuzzy 3D face race classification algorithm using a Gabor filter to differentiate between Eastern and Western people.

[19] focus on race recognition based on facial images using Principal Component Analysis (PCA) and Nearest Neighbor Classification (NNC). The study also points out that further improvement for multiple scales' inputs on a classification system. [20] come out with a local approach to 3D face recognition based on isogeodesic stripes [21] combined with minimal-redundancy maximal-relevance [22] feature selection model in order to analyse the differences of relative relevance of the facial stripes from ethnic different races.

In recent news, China uses facial recognition to monitor ethnic minorities and the system alerts authorities when targets stray beyond a "safe area" [23]. [24] used sample images from FERET database to classify three major ethnicities; Mongolian, Caucasian and Negro. Several combinations of different geometric features for different ethnicities and color attributes were extracted from the image. Artificial Neural Network and Convolution Neural Network are used for classification problem. [25] classify images into only two categories: Asian and non-Asian. They have proved that using LDA and the proposed ensemble framework, a sufficient discriminative power for the ethnicity classification problem is obtained.

[26] in their work classified face images into two race groups, Myanmar and Non-Myanmar. The NLPR database used in [27], contained face images in a variant pose, illumination, and expression. The Principal Component Analysis (PCA) based scheme has been developed for the two-class classification and $96 \%$ of accuracy reported.

\section{MUA DATABASE}

\section{A. Face Processing using Kinect}

Kinect sensor provides both colour and dense depth images. It combines structured light with depth from focus and depth from stereo. The sensor consists of infrared laserbased IR emitter, an infrared camera and a RGB camera. The IR camera and the IR projector compose a stereo pair with a baseline of approximately $75 \mathrm{~mm}$. A known pattern of dots is projected from the IR laser emitter. Since there is a distance between laser and sensor, the images correspond to different camera positions, and this, in turn, allows us to use stereo triangulation to calculate each spec depth. It captures the depth and colour images simultaneously at a frame rate of about 30 fps. The RGB stream has size $640 \times 480$ and 8-bit for each channel, whereas the depth stream is $640 \times 480$ resolution and with 11-bit depth. Depth is a very useful cue to attain reliable face detection and tracking since a face may not have consistent colour and texture but has to occupy an integrated region in space. The field of view is 57० horizontally and $43^{\circ}$ vertically. The minimum measurement range is about $0.6 \mathrm{~m}$, whereas the maximum range is somewhere between 4-5 m. Together with the sensor,, it is delivered Kinect for Windows SDK and the Face Tracking SDK, which enable developing applications capable of tracking human faces in real time. The face tracking engine determines 3D positions of semantic facial feature points as well as $3 \mathrm{D}$ head poses. It tracks the 3D location of 121 points, which are depicted in Fig. 1b. Additionally, the Face Tracking SDK fits a 3D mask to the face. The 3D model is based on the Candide 3 models [8], which is a parameterize 3D face mesh specifically developed for model-based coding of human faces. This 3D model is widely used on a head pose tracking [27].

As already indicated, Kinect sensor allows low-cost sensing with high capture speed. However, the 3D maps provided by Kinect are very noisy and have a relatively low resolution in comparison to typical devices utilized in facial expression recognition. In consequence, many important fiduciary points such as eye and mouth corners are not too precisely locatable. Even more, some fiduciary markers undergo occlusion, particularly the points that are located close to the nose.

\section{B. MUA Database Structure}

MUA database is composed of 205 subjects' facial expressions were 50 Malays, 50 Chinese, 50 Iban, 50 Indians and an addition of five subjects from other races. The total subjects include 75 males and 125 females. The subjects had to involve in two types of a data collection process: (i) spontaneous and (ii) continuous. In a spontaneous experiment, 10 videos to have been shown to the participants and the facial expressions recorded by using Kinect sensor. The videos are containing sad, happy and et cetera stories. A continuous experiment has been conducted by recording the seven types of expressions from each participant. For each expression, 3 take-ups have been recorded, which contains from low to high intensity of the facial expressions. In a continuous experiment to avoid expressions overlapping, a demo video for each expression has been shown to the participants before recording the specific expressions. Moreover, for immediate visual feedback, a mirror was placed in front of the participant. Some videos recorded with glasses, partially occluded by scarf and hair, wearing caps. Eight types of facial expressions, including neutral, angry, disgust, fear, happy, surprise, pain and sad have been recorded from all subjects.

The database consists of two groups of data: (i) 2D data set and (ii) 3D data set. The $2 \mathrm{D}$ dataset contained full RGB images, face RGB images and 2D facial points retrieved from the experiment. Whereas, 3D dataset contained 3D facial points, depth images and $3 \mathrm{D} *$.obj files processed from the facial feature points. Each of the data sets was arranged by (i) subjects, (ii) emotions and (iii) ethnics accordingly. The hierarchy of the database is best explained via Figure 1.

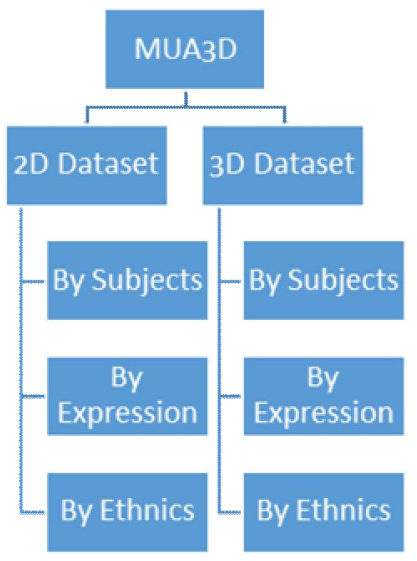

Figure 1: Hierarchy of MUA3D Database 

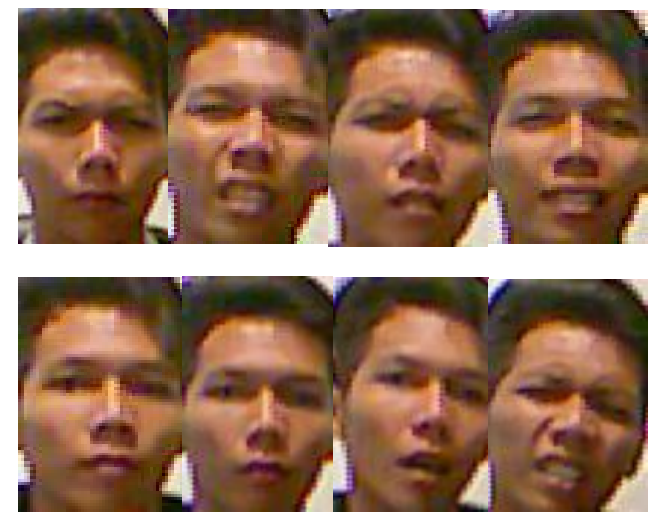

Figure 2:Facial Images for 7 Different Expressions (Angry, Disgust, Fear, Happy, Neautral, Sad and Surprise)

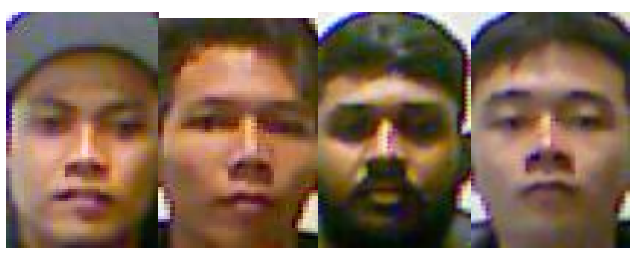

Figure 3::Facial Images for 4 Different Ethnicity (Iban, Malay, Indian and Chinese)

\section{EXPERIMENT}

\section{Experiment Setup}

The participants are fully untrained to obtained natural emotion expressions from the experiment. The subjects had to involve in two type of data collection process: (i) spontaneous and (ii) continuous. For spontaneous experiment, 10 videos to have been shown to the participants and the facial expressions recorded by using Kinect sensor. The videos are containing sad, happy and et cetera stories. The participants reported their intensity of emotions after each session of spontaneous experiments to record the ground truth of their emotion. They were given a list of six basic emotions with low, medium and high intensity and also natural.

Continuous experiment has been conducted by recording the seven types of expressions from each participant. For each expression, 3 takes have been recorded which contains from low to high intensity of the facial expressions. In continuous experiment to avoid expressions overlapping, a demo video for each expression has been shown to the participants before recording the specific expressions. Moreover, for immediate visual feedback a mirror was placed in front of the participant. Some videos recorded with glasses, partially occluded by scarf and hair, wearing caps. Eight types of facial expressions including neutral, angry, disgust, fear, happy, surprise, pain and sad have been recorded from all of the subjects.

\section{Data Processing}

From the recorded videos of each session of the experiments, continuous RGB-D images and set of 2D and 3D feature points for each frame are processed. Feature extraction processes are then performed using high intensity data. The recognition process is highly depending on the selection of feature to represent the categorized face. In this project, we have experimented the data using several features as described below.

\section{a) Gradient of Pixel}

For 2D dataset, the face images were resized to a standard resolution of $100 \times 100$ and converted into grayscale images for further processing. The images then tested using Histogram of Oriented Gradients (HOG) and compared with Local Binary Pattern (LBP) histogram.

\section{b) Euclidean Distance}

For 3D dataset, each frame contained 121 facial points in total and each facial point contained $\mathrm{X}, \mathrm{Y}$ and $\mathrm{Z}$ axis. The facial points were first aligned so that the eyes and nose tip were positioned to the same angle. The Euclidean distance of corresponding points in two adjacent frames were taken as the difference measurement. The difference of jth facial point in ith frame is calculated as Equation 1, in which,

$$
\mathrm{i} \in\{1, \cdots, \mathrm{N}-1\}, \mathrm{j} \in\{1, \cdots, 121\}
$$

So, we could get $(\mathrm{N}-1) *(121)$ matrix A. This matrix then be the features to be processed with the classifier.

\section{c) Data Classification}

The feature extraction techniques described in (a) and (b), which are HOG, LBP and Euclidean distance were carried out on the whole faces according to their categories which are by subjects, emotions and ethnics. All features are classified by Support Vector Machines (SVM) classifier for all categories.

\section{RESULT AND ANALYSIS}

\section{E. Facial Ethnicity Classification}

We have conducted experiment using our 2D image ethnicity database using HOG feature in comparison with LBP feature and classify it with SVM classifier in 10-fold cross validation for ethnicity classification between Chinese, Iban, Indian and Malay, likewise for 3D data using Euclidean Distance feature. The comparing results of accuracy, along with the success rate for each ethnicity from each different feature are shown in Table 1 below.

Table 1: Results of Accuracy and Success Rate for Each Ethnicity From Different Features

\begin{tabular}{l|c|c|c|cc}
\hline \multirow{2}{*}{ Feature } & \multirow{2}{*}{$\begin{array}{c}\text { Accuracy } \\
(\%)\end{array}$} & \multicolumn{4}{|c|}{ Success Rate (\%) } \\
\cline { 3 - 6 } & & Chinese & Iban & Indian & Malay \\
\hline HOG & 92.10 & 90.50 & 89.15 & 94.93 & 92.43 \\
\hline LBP & 50.07 & 5.87 & 89.15 & 0.97 & 3.55 \\
\hline $\begin{array}{l}\text { Euclidean } \\
\text { Distance }\end{array}$ & 37.00 & 0.00 & 0.43 & 59.26 & 66.87 \\
\hline
\end{tabular}

As can be observed, HOG features present the best accuracy results which are $92.10 \%$ when classified using SVM classifiers compared to other features, and have the best results for every ethnicity. LBP performance are just $50.07 \%$, which only have significant results for Iban ethnicity. The experiment using Euclidean Distance feature is still low in accuracy and cannot distinguish differences between ethnicity especially Chinese and Iban. This may due to the ethnicity database includes all type of emotions for each ethnicity, therefore causing confusion in measuring distance of facial points between ethnicity. 
Table 2 shows confusion matrix of ethnicity classification using HOG features and SVM classifier.

Table 2: Confusion Matrix of Ethnicity Classification Using HOG Features and SVM Classifier

\begin{tabular}{|l|c|c|c|c|}
\hline \multirow{2}{*}{$\begin{array}{c}\text { Actual } \\
\text { Class }\end{array}$} & \multicolumn{4}{|c|}{ Predicted Class (\%) } \\
\cline { 2 - 5 } & Chinese & Iban & Indian & Malay \\
\hline Chinese & 90.50 & 6.10 & 0.97 & 2.13 \\
\hline Iban & 5.87 & 89.15 & 0.97 & 3.55 \\
\hline Indian & 2.23 & 1.02 & 94.93 & 1.89 \\
\hline Malay & 1.40 & 3.73 & 3.14 & 92.43
\end{tabular}

From the table, Indians are most distinctive with accuracy of $94.93 \%$, where $3.14 \%$ are confused with Malay and $0.97 \%$ for both Chinese and Iban. It can be said that Indian have peculiar facial features compared to other. As for this experiment, it also may due to the skin intensity of the subjects' images in this groups that highlight the contrast. Malay ethnic successfully classified with accuracy $92.43 \%$ with the percentage of confusion $3.55 \%, 2.13 \%$ and $1.89 \%$ for Iban, Chinese and Indian respectively. For Chinese resulting in $90.50 \%$ accuracy, where $5.87 \%$ confused with Iban, $2.23 \%$ confused with Indian and $1.4 \%$ confused with Malay. Iban data shows the lowest accuracy value which is $89.15 \%$, with the percentage of confusion $6.10 \%$ with Chinese, $3.73 \%$ with Malay and $1.02 \%$ with Indian. From our subjects group, Iban also have the highest number from mix marriage parents which may lead for the confusion for Chinese, Indian and Malay ethnicity.

\section{F. Facial Expression Classification}

We also experimented with our 2D image and 3D database for facial expression classification. Again for this experiment, we are using HOG feature in comparison with LBP feature using the 2D images, whereas Euclidean Distance feature for 3D database, and then classify it with SVM classifier in 10-fold cross validation for expression classification between angry, disgust, fear happy, neutral, sad and surprise. From the experiment, best results come from the combination of HOG feature and SVM classifier with accuracy $59.10 \%$. The results are expected to be in average as the participants were untrained and mostly shows their own natural, un-posed expression for all emotions (angry, disgust, fear, happy, neutral, sad and surprise) when conducted the experiment. Table 3 shows the results of accuracy and success rate for each emotion from different features.

Table 3: Results of Accuracy and Success Rate for Each Emotion from Different Features

\begin{tabular}{|c|c|c|c|c|c|c|c|c|}
\hline \multirow[b]{2}{*}{ 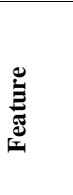 } & \multirow{2}{*}{ 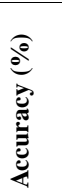 } & \multicolumn{7}{|c|}{ Success Rate (\%) } \\
\hline & & $\underset{0}{\vec{b}}$ & 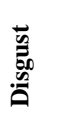 & 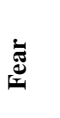 & $\frac{\overrightarrow{0}}{\bar{z}}$ & $\begin{array}{l}\bar{T} \\
\bar{\Xi} \\
Z\end{array}$ & ש్ & 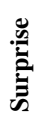 \\
\hline HOG & 59.1 & 60.4 & 67.3 & 6.92 & 94.0 & 65.4 & 41.9 & 79.5 \\
\hline LBP & 40.8 & 55.0 & 27.4 & 21.0 & 65.3 & 40.1 & 27.0 & 50.0 \\
\hline EucD & 38.1 & 16.1 & 4.80 & 9.04 & 27.1 & 93.2 & 22.6 & 66.3 \\
\hline
\end{tabular}

From the table above, HOG features again present the best results which are $59.10 \%$, followed by the results of LBP and Euclidean Distance Feature which are $40.8 \%$ and $38.10 \%$ respectively. LBP features appear to have the highest results in differentiating fear emotion, with $21.0 \%$, compared to others with only $9.04 \%$ and $6.92 \%$. Euclidean distance is observed to have the highest result in classifying neutral emotion with $93.2 \%$ and show competitive result in differentiating surprise emotion with $66.3 \%$, but fail to successfully distinguish other emotions.

Table 4 shows confusion matrix of emotion classification using HOG Features and SVM Classifier.

Table 4: Confusion Matrix of Ethnicity Classification Using HOG Features and SVM Classifier

\begin{tabular}{|c|c|c|c|c|c|c|c|}
\hline \multirow[b]{2}{*}{ 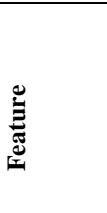 } & \multicolumn{7}{|c|}{ Success Rate (\%) } \\
\hline & $\vec{B}$ & 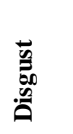 & $\underset{\mathbb{J}}{\leftrightarrows}$ & $\frac{\overrightarrow{0}}{\bar{\Xi}}$ & 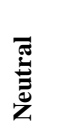 & స్ర్ర & 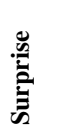 \\
\hline Angry & 60.36 & 7.10 & 3.6 & 3.6 & 9.47 & 13.61 & 2.37 \\
\hline Disgust & 5.95 & 67.27 & 4.17 & 10.71 & 1.79 & 7.74 & 2.38 \\
\hline Fear & 26.15 & 22.31 & 6.92 & 9.23 & 11.54 & 13.08 & 39.23 \\
\hline Happy & 0 & 2.40 & 0 & 94.01 & 0.60 & 1.20 & 1.80 \\
\hline Neutral & 6.17 & 0.62 & 4.32 & 6.79 & 65.43 & 8.64 & 5.56 \\
\hline Sad & 20.36 & 8.98 & 5.99 & 4.79 & 14.37 & 41.92 & 3.59 \\
\hline Surprise & 2.41 & 1.81 & 3.01 & 7.83 & 4.22 & 1.20 & 79.52 \\
\hline
\end{tabular}

From the table we can see that Happy is the most distinctive emotion with $94.01 \%$ success rate, followed by Surprise, Disgust, Neutral and Angry with 79.52\%, 67.27\%, $65.43 \%$ and $60.36 \%$ respectively among the high success rate. As expected, happy and surprise appear to be among the highest in accuracy as the two expressions are the most frequently used in communication and therefore quickly identified compared to others [30]. Whereby, angry, disgust, sad and fear expression appear to be difficult to classify as all four are categorized as negative emotions which are often difficult to be classified [31]. They are also observed to occasionally appear in combinations, in which one of them shows higher intensity [31]. Sad and Fear appear to be among the lowest success rate with $41.92 \%$ and $6.92 \%$ respectively. As for Fear, the biggest confusion for this experiment appears to occur with Surprise, which can be explained from [32], the difficulty in recognising fear may account for the similar visual configuration with surprise. As for sad, it often appear as a micro-expressions, which are occur very brief especially for people who done it spontaneously or without experience in acting the emotions like the subjects for this experiment. This also means that usually people hard to express their sadness and in other hand causing people hard to detect this sadness. Therefore the result for sadness is less adequately recognized and show strong asymmetric confusion with the others, especially angry and neutral. However, neutral and anger expression are rarely confused for sadness. 
In other hand, we may observe the results in correspond to the AUs for each emotion. [33] concluded that surprise usually includes the activation of AU $1+2+5+25$. Note that fear activates AU $1+5+25$, which are subsets of those of surprise. Therefore fear is confused as surprise and not the other way round. Same as occurred for sad which includes AU $1+4+15$. If the AU 15 is not notable, which in [32] only 0.98 AU evidence scores of significance for sad, it can be confused with angry and disgust for the existence of AU 4 (with AU evidence score of 1.55 for sad) and also fear and surprise for the presence of AU 1 (with AU evidence score of 1.38 for sad). Unlike happy, even though it only includes AU $6+12+25$, each AU have 2.88, 4.06 and 2.07 evidence scores respectively, makes its easily distinguished compared to other emotions. As for anger in other hand, with AU $4+7$, have risk to be confused with disgust (AU $4+6+7+9+$ $10)$ as it is subset for the expression, and also sad if the AU 7 (with AU evidence score of 0.89 for anger) is not observable.

\section{G. Face Recognition}

The 2D image databases are also tested for facial recognition. In this experiment, we are using HOG feature in comparison with the LBP feature and classify it with SVM classifier in 10-fold cross validation for all participants. The best results are from HOG feature with SVM classifier with accuracy $94.10 \%$, which are quite competitive to existing benchmark databases.

\section{CONCLUSION}

We introduce MUA, a Malaysian ethnicity database for various visual computational applications. The database composed of 205 subjects' facial expressions, which are angry, disgust, fear, happy, neutral, sad and surprise, where 50 are Malays, 50 are Chinese; 50 are Iban; 50 are Indian and an additional of 5 subjects from other races. The database consists of two groups of data: (i) 2D data set, which contained of full RGB images, face RGB images and 2D facial points and (ii) 3D data set, contained 3D facial points, depth images and 3D*.obj files processed from the facial feature points. The dataset was used in the experiments for facial ethnicity classification and facial expression classification with a percentage of accuracy $92.10 \%$ and $59.10 \%$ respectively. We foresee various other applications benefiting from MUA database forthcoming.

\section{ACKNOWLEDGMENT}

This research is fully supported by Malaysian Ministry of Higher Education (MOHE) through Race Acculturation Collaborative Efforts RACE/1331/2016(4). The authors fully acknowledged MOHE and Universiti Malaysia Sarawak for the approved fund which makes this important research viable and effective.

\section{REFERENCES}

[1] A. J. Calder, G. Rhodes, M. Johnson, and J. V. Haxby, Oxford Handbook of Face Perception. London, U.K.: Oxford Univ. Press, 2011.

[2] J. L. Eberhardt, N. Dasgupta, and T. L. Banaszynski, "Believing is seeing: The effects of racial labels and implicit beliefs on face perception," Personality Soc. Psychol. Bull., vol. 29, pp. 360-412, 2003. https://doi.org/10.1177/0146167202250215

[3] J. Freeman, N. Ambady, and P. Holcomb, "The facesensitive n170 encodes social category information," NeuroReport, vol. 21, no. 1, pp. 24-28, 2010.

[4] S. Bentin and L. Y. Deouell, "Structural encoding and identification in face processing: ERP evidence for separate mechanisms," Cogn. Neuropsychol., vol. 17, pp. 35-54, 2000.

[5] B. Rossion and C. Jacques, "N170: Understanding the time course of face perception in the human brain," Int. J. Neurosci., vol. 112, pp. 1499-1512, 2002.

[6] R. Kurzban, J. Tooby, and L. Cosmides, "Can race be erased? Coalitional computation and social categorization," Proc. Nat. Acad. Sci., vol. 98, no. 26, pp. 15387-15392, 2001.

https://doi.org/10.1073/pnas.251541498

[7] P. Phillips, P. Flynn, T. Scruggs, K. Bowyer, J. Chang, K. Hoffman, J. Marques, J. Min, and W. Worek, "Overview of the face recognition grand challenge," in Proc. IEEE Conf. Comput. Vis. Pattern Recog., 2005, pp. 947-954.

[8] P. Phillips, W. Scruggs, A. OToole, P. Flynn, K. Bowyer, C. Schott, and M. Sharpe, "FRVT 2006 and ICE 2006 largescale experimental results," IEEE Trans. Pattern Anal. Mach. Intell., vol. 32, no. 5, pp. 831-846, May 2010.

[9] K. Bowyer, K. Chang, and P. Flynn, "A survey of approaches and challenges in 3D and multi-modal $3 \mathrm{D}+2 \mathrm{D}$ face recognition," Comput. Vis. Image Understanding, vol. 101, no. 1, pp. 1-15, 2006.

https://doi.org/10.1016/j.cviu.2005.05.005

[10] K. Chang, K. Bowyer, and P. Flynn, "An evaluation of multimodal 2D+3D face biometrics," IEEE Trans. Pattern Anal. Mach. Intell., vol. 27, no. 4, pp. 619-624, Apr. 2005.

[11] D. T. Levine and B. L. Angelone, "Categorical perception of race," Perception, vol. 31, pp. 567-578, 2002.

[12] D. T. Levine, "Classifying faces by race: The structure of face categories," J. Exp. Psychol.: Learn., Memory Cogn., vol. 22, pp. 1364-1382, 1996.

[13] D. T. Levine, "Race as a visual feature: Using visual search and perceptual discrimination tasks to understand face categories and the cross-race recognition deficit," J. Exp. Psychol.: Gen., vol. 129, pp. 559-574, 2000.

[14] Ford, M. E., \& Kelly, P. A. (2005). Conceptualizing and categorizing race and ethnicity in health services research. Health services research, 40(5p2), 1658-1675.

[15] D. A. Wilbraham, J. C. Christensen, A. M. Martinez, and J. T. Todd, "Can low level image differences account for the ability of human observers to discriminate facial identity?" J. Vis., vol. 8, no. 15, p. 5, 2008.

https://doi.org/10.1167/8.15.5

[16] L. G. FARKAS, Anthropometry of the Head and Face., NY: Raven Press, New York, 1994

[17] Lu, Xiaoguang and Chen, Hong and Jain, Anil K, "Multimodal facial gender and ethnicity identificatio," International Conference on Biometrics, pp. 554-561, 2006.

[18] Zhong, Cheng and Sun, Zhenan and Tan, Tieniu, "Fuzzy 3D face ethnicity categorization," International Conference on Biometrics, pp. 386-393, 2009.

[19] Tin, H. H. K., \& Sein, M. M, "Automatic Race Identification from Face Images in Myanmar," The First International Conference on Energy Environment and Human Engineering, 2013.

[20] Berretti, Stefano and Del Bimbo, Alberto and Pala, Pietro, "Distinguishing facial features for ethnicitybased 3D face recognition," ACM Transactions on Intelligent Systems and Technology (TIST), p. 45, 2012.

[21] Peng, H., Long, F., \& Ding, C. "Feature selection based on mutual information criteria of max-dependency, maxrelevance, and min-redundancy," IEEE Transactions on pattern analysis and machine intelligence, 27(8), p. 1226-1238, 2005.

[22] Berretti, Stefano, Alberto Del Bimbo, and Pietro Pala. "Description and retrieval of 3D face models using isogeodesic stripes." Proceedings of the 8th ACM 
international workshop on Multimedia information retrieval. ACM, 2006.

[23] https://www.engadget.com/2018/01/18/china-facialrecognition-uyghurs-xinjiang. Last accessed on 17 th August 2018.

[24] Masood S., Gupta, S, Wajid, A, Gupta, S, and Ahmed, M. Prediction of Human Ethnicity from Facial Images Using Neural Networks. In: Satapathy SC, Bhateja V, Raju KS and Janakiramaiah B. Data Engineering and Intelligent Computing. Singapore: Springer. 2018: $217-226$.

[25] Lu, Xiaoguang \& K. Jain, Anil. Ethnicity Identification from Face Images. Proc SPIE. 2004. 5404. $10.1117 / 12.542847$.

[26] H. H. K. Tin and M. M. S. Race Identification for Face Images. ACEEE International Journal on Information Technology, Vol. 01, No. 02.

[27] Kwolek, B.: Model based facial pose tracking using a particle filter. In: Int. Conf. on Geometric Modeling and Imaging: New Trends, IEEE Comp. Soc. (2006) 203208.

[28] S. Du and A. M. Martinez, "The resolution of facial expressions of emotion," Journal of Vision, vol. 11, no. 13 , p. 24, 2011.

[29] S. S. Kulkarni et. al., "Facial expression (mood) recognition from facial images using committee neural networks," Biomedical engineering online, vol. 8, no. 1, p. 16, 2009.

[30] Roy-Charland, A., Perron, M., Beaudry, O., \& Eady, K., "Confusion of fear and surprise: A test of the perceptual-attentional limitation hypothesis with eye movement monitoring," Congnition and Emotion, vol. 7 , no. 28, pp. 1214-1222, 2014.

[31] M. G. Calvo et. al., "Human observers and automated assessment of dynamic emotional facial expressions: KDEF-dyn database validation," Frontiers in Psychology, vol. 9, p. 2052, 2018. 